\title{
Photometric and TiO modelling of the starspots on AG Dor and HU Vir
}

\author{
P. J. Amado ${ }^{1}$ and M. Zboril ${ }^{2, \star}$ \\ 1 Instituto de Astrofísica de Andalucía-CSIC, Apartado 3004, 18080 Granada, Spain \\ 2 Astronomical Institute, Tatranská Lomnica, 059 60, Slovakia
}

Received 2 May 2001 / Accepted 19 October 2001

\begin{abstract}
In this work, spot parameters are determined for two stars, namely, AG Dor and HU Vir, from their photometric variability and $\lambda 7055 \AA \mathrm{TiO}$ band depth. Applying the photometric modelling code SPOTPIC, we have found two modulating spots together with a non-modulating component on the photosphere of AG Dor in December 96/January 97. The spot temperatures are constrained to be between $4300 \mathrm{~K}$ and $4600 \mathrm{~K}$ for the modulating component, and cooler, viz., $4000 \mathrm{~K}$, for the non-modulating one. Therefore, the non-modulating component of the spot distribution on AG Dor at this epoch is held to be totally responsible for the presence of the $\mathrm{TiO}$ band in the spectra of this K1 dwarf with photospheric temperature of $5000 \mathrm{~K}$. AG Dor's total coverage of spots is determined to be between $4 \%$ and $7 \%$ for the modulating component and $26.5 \%$ for the non-modulating one. For HU Vir, the light and colour curves were modelled with two spots with temperatures of 4450 and $4050 \mathrm{~K}$. In this case we did not include the non-modulating component in the model.
\end{abstract}

Key words. stars: activity - stars: late-type - stars: variables - stars: individual: AG Dor - stars: individual: HU Vir

\section{Introduction}

Many stars show variations in their $V$-band light curve which can be accounted for by assuming that cool magnetic parts of the stellar photosphere (starspots) are carried on, across and off the stellar disk by the star's rotation. These variations in light can also be seen in colour indices like $(V-R)_{\mathrm{c}},(V-I)_{\mathrm{c}}$ or $(V-K)$, usually in phase with the $V$ curve, showing a reddening at light minimum (maximum spot visibility) and, thus, supporting the cool spot hypothesis.

Further evidence of such a hypothesis comes from the fact that some spectral features, especially molecular bands (e.g. TiO bands), characteristic of the cooler spots, are visible at some or even at all phases throughout the rotation of the star. The presence of these bands reveals the existence of these spots since at the effective temperature of the unspotted photosphere, none should be seen.

In the past decade, there has been a significant advance in the number of methods that are available to study starspots. The temperature sensitivity of some molecular bands, like titanium oxide (TiO), carbon monoxide (CO) and hydrogen monoxide $(\mathrm{OH})$, has been used to derive effective temperatures and areas of spots (Neff et al. 1992; Neff et al. 1995; O'Neal et al. 1996). Indirect (Doppler)

Send offprint requests to: P. J. Amado, e-mail: pja@iaa.es

* Present address: Astrophysikalisches Institut, Potsdam, An der Sternwarte 16, Potsdam 14482, Germany imaging techniques have opened up a whole new discipline in stellar astronomy, providing increasingly detailed photometric and magnetic images of stellar surfaces (see the review by Strassmeier 2001 and references therein). New optical interferometers are already being used with sophisticated interferometric techniques to image stellar surface structures directly (von der Lühe et al. 1996). Complementing all these new procedures, older methods, e.g., the broad-band photometric technique, are still indispensable in constraining possible surface map solutions.

$U B V(R I)_{\mathrm{c}}, J H K$ photometry of 2 active stars and $\lambda 7055 \AA \mathrm{TiO}$ band spectroscopy of one of them were collected at the South African Astronomical Observatory in 1996 and 1997 and presented in Amado et al. (2001) (hereafter Paper I). In Paper I, the light and colour curves were compared with those observed at previous epochs, showing significant variations of the wave-like modulation and of their maximum brightness.

We will use the broad-band photometry and $\mathrm{TiO}$ spectroscopy to derive the spot distribution on a set of active stars. To model the light and colour curves of these stars we have used the theoretical code SPOTPIC, developed by one of us. For a description of this code see Amado et al. (1999) and Amado et al. (2000). In order to be able to employ the $\mathrm{TiO}$ technique to derive the physical parameters of the spots, a number of inactive $\mathrm{G}, \mathrm{K}$ and $\mathrm{M}$ stars were also observed spectroscopically ( $\mathrm{TiO}$ band). 
The simultaneity of these photometric and spectroscopic observations permitted both techniques, namely, the light-curve modelling technique and the TiO band technique, to be compared.

\section{Data}

The photometric data were collected at the South African Astronomical Observatory (SAAO) during the week of January 30 to February 6 in 1996. In 1997, the observations were taken between December 241996 and January 6 1997 and between January 23 and February 3. The same telescopes and instrumental configurations were used in the 1996 and 1997 runs. For more details on these data see Paper I.

The spectroscopic data were collected at the SAAO with the $1.9 \mathrm{~m}$ telescope between December 241996 and January 6 1997. Tables 1 and 2 list the program stars observed photometric and spectroscopically at the SAAO. The data for the standard stars are listed in Table 3 . The spectrograph configuration, set with grating number 9, permitted a useful range of $1100 \AA$ around a central wavelength of $\sim 7100 \AA$, which left out the $\lambda 8860 \mathrm{TiO}$ band, but included $\mathrm{H} \alpha$. These data were flat-field corrected, sky subtracted, wavelength calibrated and continuum normalized using IRAF $^{1}$ (Tody 1986). The wavelength calibration was achieved by collecting arc spectra from a Copper-Argon lamp, with measurements of arc lines giving an instrumental resolution of $\sim 1.7 \AA$. The data were also corrected for the presence of telluric lines. In order to do so, rapidly rotating $\mathrm{B}$ and $\mathrm{A}$ stars were observed as close in time and on the sky to our target stars as possible. Previously, the telluric lines were used to bring all the spectra to the same wavelength reference system.

\section{Photometric technique}

Basically, SPOTPIC is a FORTRAN code developed to compute synthetic light and colour curves of active latetype stars with temperature inhomogeneities on their surfaces. It uses the effective temperature-colour relationships derived by Amado (1997) and the calibration of the surface brightness parameter $F_{V}$ against the $\left(I_{\mathrm{c}}-K\right)$ colour index from Amado et al. (1999) to compute the colours and flux ratios, respectively, of the spotted and unspotted photosphere of the star. The code calculates, by direct integration on the visible surface of the star, the projected area of a determined number of spots and finds the best fit to the observed light and colour curves simultaneously in $V,(V-R)_{\mathrm{c}},(V-I)_{\mathrm{c}}$ and $(V-K)$ by means of the Levenberg-Marquardt least-square minimization procedure.

\footnotetext{
${ }^{1}$ IRAF is distributed by the National Optical Astronomy Observatories (NOAO), which are operated by the Association of Universities for Research in Astronomy, Inc., under cooperative agreement with the National Science Foundation.
}

Table 1. Stars observed at SAAO.

\begin{tabular}{|lrrcc|}
\hline \multicolumn{1}{|c}{$\begin{array}{c}\text { Program } \\
\text { Star }\end{array}$} & $V_{\max }$ & $P_{\text {phot }}$ & Sp. T. & SB \\
\hline AG Dor & 8.67 & 2.533 & K1Vp & 2 \\
HU Vir & 8.57 & 10.28 & K0IV & 1 \\
V1005 Ori & 10.05 & 4.40 & dM0e & 1 \\
CD -28 2525 & 9.05 & 2.70 & G1V & 1 \\
YZ CMi & 12.29 & 2.78 & dM4.5e & 1 \\
TY Pyx & 6.835 & 3.32 & G5IV /G5IV & 2 \\
\hline
\end{tabular}

Table 2. Active stars observed spectroscopically in January 1997.

\begin{tabular}{|lccc|}
\hline \multicolumn{1}{|c}{ Star } & Spectral Type & $V$ & $(B-V)$ \\
\hline AG Dor & K1 Vp & 8.724 & 0.961 \\
HU Vir & K0 IV & 8.727 & 1.021 \\
CD $-28^{\circ} 2525$ & G1 V & 9.057 & 0.614 \\
EI Eri & G5 IV & 7.077 & 0.712 \\
\hline
\end{tabular}

Table 3. Comparison stars and measured $\lambda 7055 \mathrm{TiO}$ band depths.

\begin{tabular}{|crcccr|}
\hline HD & \multicolumn{1}{c}{ HR } & Spectral Type & $V$ & $(B-V)$ & $D_{7055}{ }^{1}$ \\
\hline 19826 & 953 & K0 III & 6.37 & 0.93 & 1.4 \\
33555 & 1685 & K1 IV & 6.23 & 0.98 & - \\
29503 & 1481 & K1 III & 3.87 & 1.09 & - \\
20720 & 1003 & M3/4 III & 3.65 & 1.61 & 43.0 \\
19285 & - & M5 III & - & - & 65.3 \\
\hline
\end{tabular}

${ }^{1}$ Band depth index, in percent of continuum level.

\section{TiO band technique}

The idea that bands of titanium oxide (TiO) could be used to measure starspot properties was first stated by Vogt (1979) and by Ramsey \& Nations (1980). The photometric technique cannot be used to measure the total area of spot coverage, unless the unspotted light level of the star is known. Doppler imaging has also its limitations; for example, it can only measure spot filling factors for rapidly rotating stars. The $\mathrm{TiO}$ band technique, on the other hand, can detect absolute values of the spot coverage on slow rotating stars with uniform spot coverage.

In order to be able to measure absolute values for the spot areas, Neff and collaborators (Neff et al. 1992; Neff et al. 1995; O'Neal et al. 1996) used the idea proposed by Vogt (1979) and Ramsey \& Nations (1980). They extended it by making use of multiple molecular bands with different temperature sensitivity to independently measure spot areas and temperatures. They modelled the spectral regions around the $\mathrm{TiO}$ molecular bands at $7055 \AA$ and $8860 \AA$ of active stars with combinations of inactive stars from a complete grid of $\mathrm{G}, \mathrm{K}$ and $\mathrm{M}$ proxy stars. They used the spectra of the inactive $\mathrm{G}$ and $\mathrm{K}$ stars to model the unspotted photosphere of active stars and inactive $\mathrm{M}$ stars to model the effect of the spotted regions on these active stars. These proxy spectra were then weighted by their relative continuum fluxes and by a surface area covering factor to reproduce the spectra of the active stars. 
So, they were able to measure the effective temperatures and area coverage of starspots on active stars using the equation,

$F_{\text {total }}=\frac{f_{\mathrm{s}} R_{\lambda} F_{\mathrm{s}}+\left(1-f_{\mathrm{s}}\right) F_{\mathrm{Q}}}{f_{\mathrm{s}} R_{\lambda}+\left(1-f_{\mathrm{s}}\right)}$

where $F_{\text {total }}, F_{\mathrm{s}}$ and $F_{\mathrm{Q}}$ are, respectively, the active, spot comparison and quiet comparison star spectra, $f_{\mathrm{s}}$ is the total fractional projected area of spots on the visible hemisphere and $R_{\lambda}$ is the continuum surface flux ratio between the spot and the quiet photosphere.

\section{Results}

\subsection{AG Dor (HD 26354)}

AG Dor (HD 26354) is a non-eclipsing, double-lined spectroscopic binary (SB2) (Washüttl \& Strassmeier 1995). From an extensive high resolution spectroscopic study, these last authors deduced projected rotational velocities, $v \sin i$, of $17 \pm 2$ and $10 \pm 5 \mathrm{~km} \mathrm{~s}^{-1}$ for the primary and secondary components respectively. These values translate to minimum radii of 0.86 and $0.51 R_{\odot}$, strengthening the BY Dra classification of $\mathrm{K} 1 \mathrm{~V}+\mathrm{K} 5 \mathrm{~V}$, and minimum inclination angles of the rotational axes of $\simeq 60^{\circ}$ and $\simeq 73^{\circ}$ for the components. For such a system, the maximum value of the inclination angle for which no eclipses are observed is $\simeq 80^{\circ}$, restricting the range of possible inclination angles between 73 and 80 degrees, if we assume them to be the same for both components.

\subsubsection{Photometric model}

In order to determine the parameters of the spot distribution on AG Dor during the 1997 epoch, we modelled its light and colour curves with the FORTRAN code SPOTPIC by assuming two circular spots for the modulating component and another one, placed at the pole, for the non-modulating one. Two spots were included in the models to represent the modulating component because there seemed to be some indication of structure in the minima of the $V,(V-I)_{\mathrm{c}}$ and, especially, $(V-K)$ curves (see Fig. 5).

The physical parameters of the star were derived from its spectral type and luminosity and, therefore, assumed to have a photospheric effective temperature $T_{\mathrm{ph}}=5000 \mathrm{~K}$ and a photospheric surface gravity $\log g_{\mathrm{ph}}=5.0$, while its inclination was set to $i=75^{\circ}$.

Three other models were computed for comparison, differing from the one mentioned above in the assumed values of $\log g_{\mathrm{ph}}$ and $\log g_{\mathrm{sp}}$ (effective gravity of the spots; see Amado et al. 2000 for a justification of this assumption) and $T_{\mathrm{ph}}$. The spot parameters are given in Table 4 for the four different models. Models 1 and 2 are represented in Fig. 5 by the solid thick and thin lines whereas models 3 and 4 are represented by the dashed thick and thin lines.
Table 4. Spot parameters for AG Dor. The $\chi^{2}$ of the models is relative to Model 1.

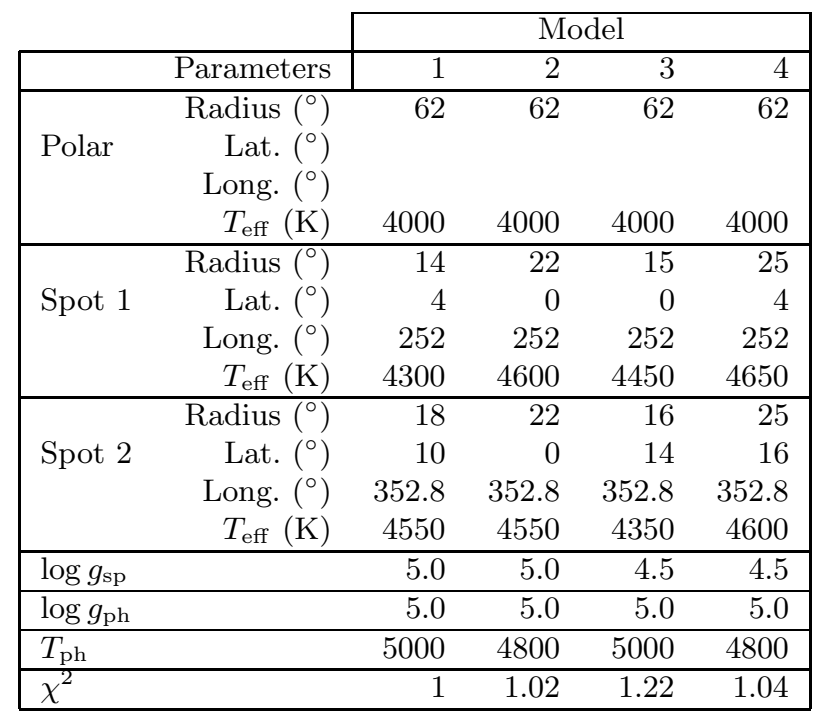

The zero level of the magnitudes for the unspotted surface were taken from Cutispoto (1996), where the star was observed in February-March 1990, and the magnitudes appeared brighter, at light maximum, than in any previous epochs. Note, however, that this does not mean the star had no spots on its surface at that epoch, which, if it did, would have affected the modelling of the nonmodulating component in the data presented here. If there were spots on the surface, dimming the maximum brightness of the star at that epoch, our non-modulating spot solution does not represent the total surface area covered by this component. The $\mathrm{TiO}$ band technique, as already mentioned, can detect absolute values of the spot coverage of uniform distribution. All this applies to the other star studied in this work.

The best fit to the data is given by Model 1. The other three models also give reasonably good fits but the data is not of sufficient quality to distinguish between them. The range in temperature given by all the models for the modulating component is between 4300 and $4650 \mathrm{~K}$. It is interesting to note that the temperature of the nonmodulating component is much lower, i.e. $4000 \mathrm{~K}$, than of the modulating one. AG Dor's total coverage of spots is determined to be between $4 \%$ (Model 1) and 7\% (Model 2) for the modulating component and $26.5 \%$ for the nonmodulating one.

Figure 1 represents four different views of the star with its modulating spot-component at angles of 45, 135, 225 and 315 degrees. The colour of the spots in the figure does not show their modelled temperature, and the rotational axis of the star should be lowered to $75^{\circ}$ to represent the actual morphology of the star. 

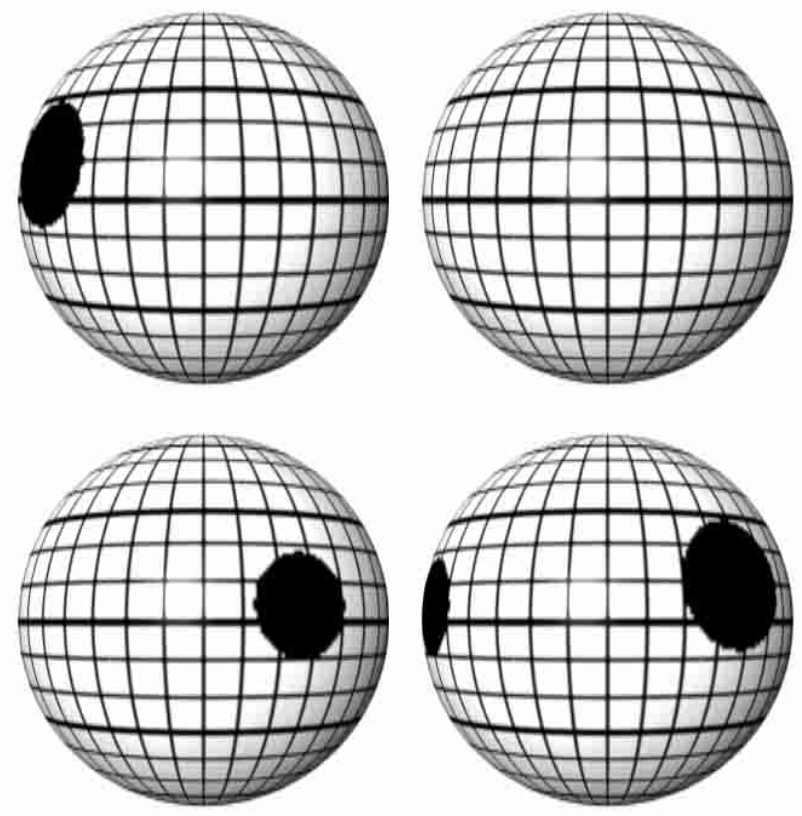

Fig. 1. AG Dor, rotating from right to left, at 45 (upper left), 135 (upper right), 225 (lower left) and 315 (lower right) degrees. Here, only the position on the surface of the star, but not the temperature, of the modulating component of the spot distribution is represented. The grid gives differences in latitude and longitude of $10^{\circ}$ with the equator and the $30^{\circ}$ and $60^{\circ}$ latitude circles emphasized over the rest.

\subsubsection{Effective temperature calibrations from $\mathrm{TiO}$ band measurements}

The band depths listed in Table 3 for the inactive comparison stars are plotted in Fig. 2 (solid triangles) along with the data from Neff et al. (1995) and O'Neal et al. (1996) (open squares). The measurements of the $\mathrm{TiO}$ band-head depth were made in two $5 \AA$-wide regions to the blue $(\lambda \lambda 7043-7048 \AA)$ and red $(\lambda \lambda 7060-7065 \AA)$ of the bandhead as in Neff et al. (1995). It can be seen that the three standard star band depths measured here fall on top of O'Neal et al.'s (1996) data. It is apparent that, for the $7055 \AA$ band, there seems to be a levelling off of the depth of the band for the coolest stars, which could be due to the TiO absorption beginning to saturate. Usually, another $\mathrm{TiO}$ band at $\lambda 8860 \AA$ is also recorded in order to uniquely determine effective temperature and areas, but this was not possible here because of the low sensitivity of the instrument at those wavelengths.

\subsubsection{Application of the technique to AG Dor}

In Fig. 3, the spectra recorded on each clear night over the region around the $\mathrm{TiO}$ band at $\lambda 7055 \AA$ are shown. The uppermost spectrum is an overall mean of the nightly spectra. On these spectra, measurements of the TiO bandhead depth were taken as explained above, and the results plotted against phase together with the $V$ light curve. No rotational modulation of the band depth was found

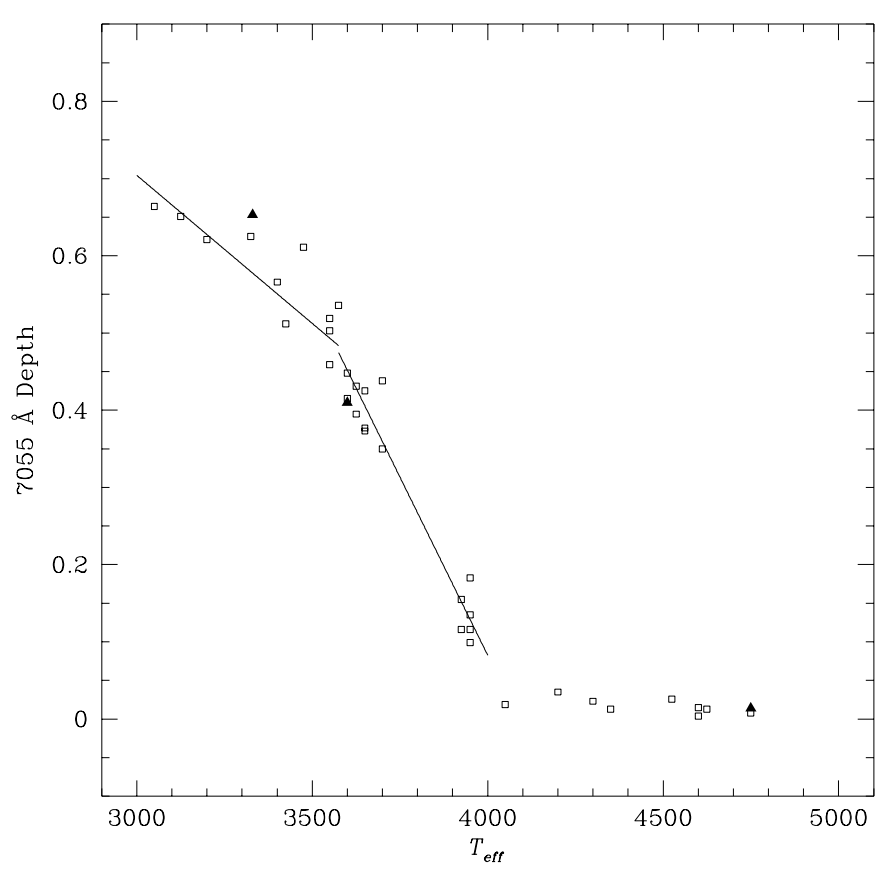

Fig. 2. Plot showing the $7055 \AA$ band depth vs. $T_{\text {eff }}$ for observed $\mathrm{K}$ and $\mathrm{M}$ giant comparison stars from O'Neal et al. (1996). Their data are shown by the open squares, with the solid lines being the best fits, and the data presented in this work by the solid triangles.

within the errors probably due to the low temporal and spectral resolution of our data.

The observed spot standard star spectra (HR 1003, M3-4 III and HD 19285, M5 III) were used to synthesize the spectra of K5 III, M1 III and M7 III stars, since not enough standard stars were observed due to bad weather. This was done by computing the band depth of the synthetic spectrum using its effective temperature (from Schmidt-Kaler 1982) and the band depth- $T_{\text {eff }}$ calibrations given by Eq. (2) in the paper by O'Neal et al. (1996). Then, the spectrum of the nearest, in spectral type, observed standard star was multiplied by a scaling factor to bring its band depth to the value of the synthetic one, restoring the continuum to its original position of 1 . When we synthesized the K5 III spectrum, the scaling factor (0.198) was so small that the noise and photospheric lines in the spectrum were lost. To correct for this effect, this spectrum was multiplied by the standard K1 III spectrum in order to add some noise and the photospheric lines. The resulting standard spectra are plotted in Fig. 4.

Once this was done, these data were used to simulate spot spectra. The star HR 953 (K0 III) was used as the model for the unspotted photosphere of AG Dor. A better model would have been provided by either a star of spectral type $\mathrm{K} 1 \mathrm{~V}$ or the K1 IV star HR 1685 but no star of luminosity class $\mathrm{V}$ was observed and the spectrum of HR 1685 was too noisy.

The excess absorption in AG Dor is weak but clearly present. To model its mean spectrum, Eq. (1) was used, where the continuum flux ratio $R_{\lambda}$ between the spotted 


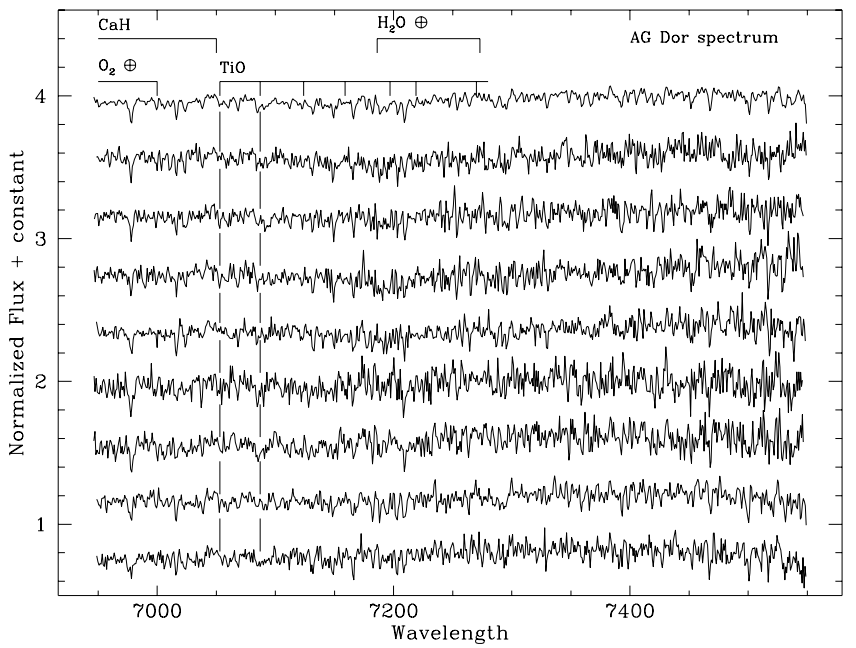

Fig. 3. AG Dor's nightly spectra in the region of the $\mathrm{TiO}$ molecular band. The spectra have been normalized to the local continuum and shifted for clarity. The spectrum at the top is the overall mean for all the nights. The TiO (especially the $\lambda 7053)$ and $\mathrm{CaH}$ molecular bands and the telluric $\mathrm{O}_{2}$ and $\mathrm{H}_{2} \mathrm{O}$ have been marked.

and unspotted photosphere was computed from the $T_{\text {eff }}-$ $\left(I_{\mathrm{c}}-K\right)$ relationship derived by Amado (1997).

Varying the filling factor and the temperature of the spots, we have fitted the resulting simulated spectra to the observed ones. Since only one band head was observed, it was not possible to constrain the temperature and the filling factor of the spots simultaneously from the spectroscopic data. Also, due to the weakness of the band and to the low resolution of the data, it was not clear which of the spectra simulated by changing the size of a spot with a temperature of $\sim 4000 \mathrm{~K}$ fitted the observed spectrum best. The best fits to the observed $\mathrm{TiO}$ spectroscopic data yield values for the spot filling factor and temperature around those of the non-modulating component of the photometric solution. This supports the conclusion that, since at the temperature of the "photometric" modulating spots (see Table 4) their contribution to the depth of the $\mathrm{TiO}$ band is probably negligible, the non-modulating component of the spot distribution, which is cooler than the modulating one, must be responsible for the presence of the $\lambda 7055 \AA \mathrm{TiO}$ band.

\subsection{HU Vir (HD 106225)}

HU Vir (HD 106225) is a SB1 K1 subgiant (Strassmeier 1994; Cutispoto 1998). It presents a photometric period of 10.28 days (Fekel et al. 1984). HU Vir also shows Ca II H and $\mathrm{K}$ very strongly in emission (Montes et al. 1996), $\mathrm{H} \alpha$ and ultraviolet emission (Fekel et al. 1986), coronal X-ray (Dempsey et al. 1993) and radio emission (Drake et al. 1989) and spectral line variations (Strassmeier et al. 1990). For a detailed study of its physical parameters see Strassmeier (1994).

In Fig. 5, the adopted spot model for the 1997 data is shown by the solid line. The sharp peak is difficult to

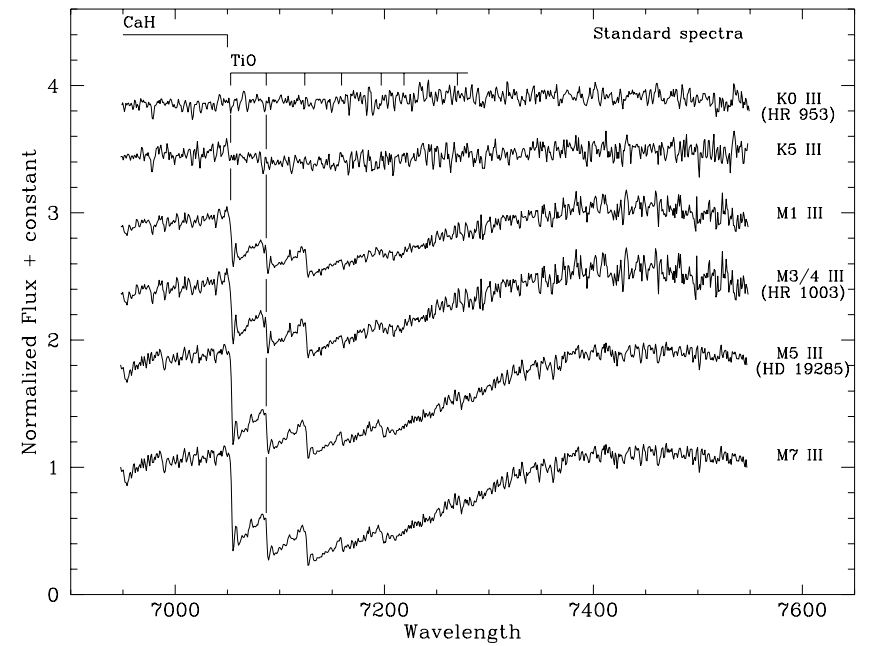

Fig. 4. Normalized standard star spectra recorded (with the name of the star between brackets) and synthesized to be used as proxies for AG Dor's photospheric (the K0III star, HR 953) and spot spectra. The spectra have been shifted and the $\lambda 7055 \AA \mathrm{TiO}$ and $\lambda 6750 \AA \mathrm{CaH}$ molecular bands marked for clarity.

Table 5. Spot parameters for HU Vir.

\begin{tabular}{|l|rr|}
\hline & Spot 1 & Spot 2 \\
& & \\
& & \\
& & \\
Radius $\left(^{\circ}\right)$ & 40 & 60 \\
Lat. $^{\circ}{ }^{\circ}$ & 55 & 11 \\
Long. $\left(^{\circ}\right)$ & 151 & 950 \\
$\Delta T(\mathrm{~K})$ & 550 & \\
& \multicolumn{2}{|c|}{3.5} \\
\hline $\log g_{\mathrm{sp}}$ & \multicolumn{2}{|c|}{4.0} \\
\hline $\log g_{\mathrm{ph}}$ & \multicolumn{2}{|c|}{5000} \\
\hline$T_{\mathrm{ph}}$ & \\
\hline
\end{tabular}

reproduce and the gaps in the data between phases 0.0 and 0.4 in the optical and the sparse data in the infrared complicates the study. However, it was obvious that, in order to fit simultaneously the optical and the infrared colours, a cooler temperature and, therefore, smaller size for the spots was necessary than when only fitting the optical colours. In Table 5 , the parameters of the spots used in the fit are listed, while the physical parameters of the star were taken from Strassmeier (1994), namely, $T_{\mathrm{ph}}=5000 \mathrm{~K}$; $\log g_{\mathrm{ph}}=4.0$ and $i=65^{\circ}$. The effective gravity of the spots $\left(\log g_{\mathrm{sp}}\right)$ was set to 3.5 . The zero points for the magnitudes of the unspotted photosphere were set to the light and colour curves' maxima, i.e., the non-modulating component was not included in the model. We preferred not to model this component because of the poorly defined maximum brightness of the $(V-K)$ colour curve. However, the star was close to its historical maximum brightness (Strassmeier et al. 1997) and, therefore, this component must have been very small and difficult to model within 

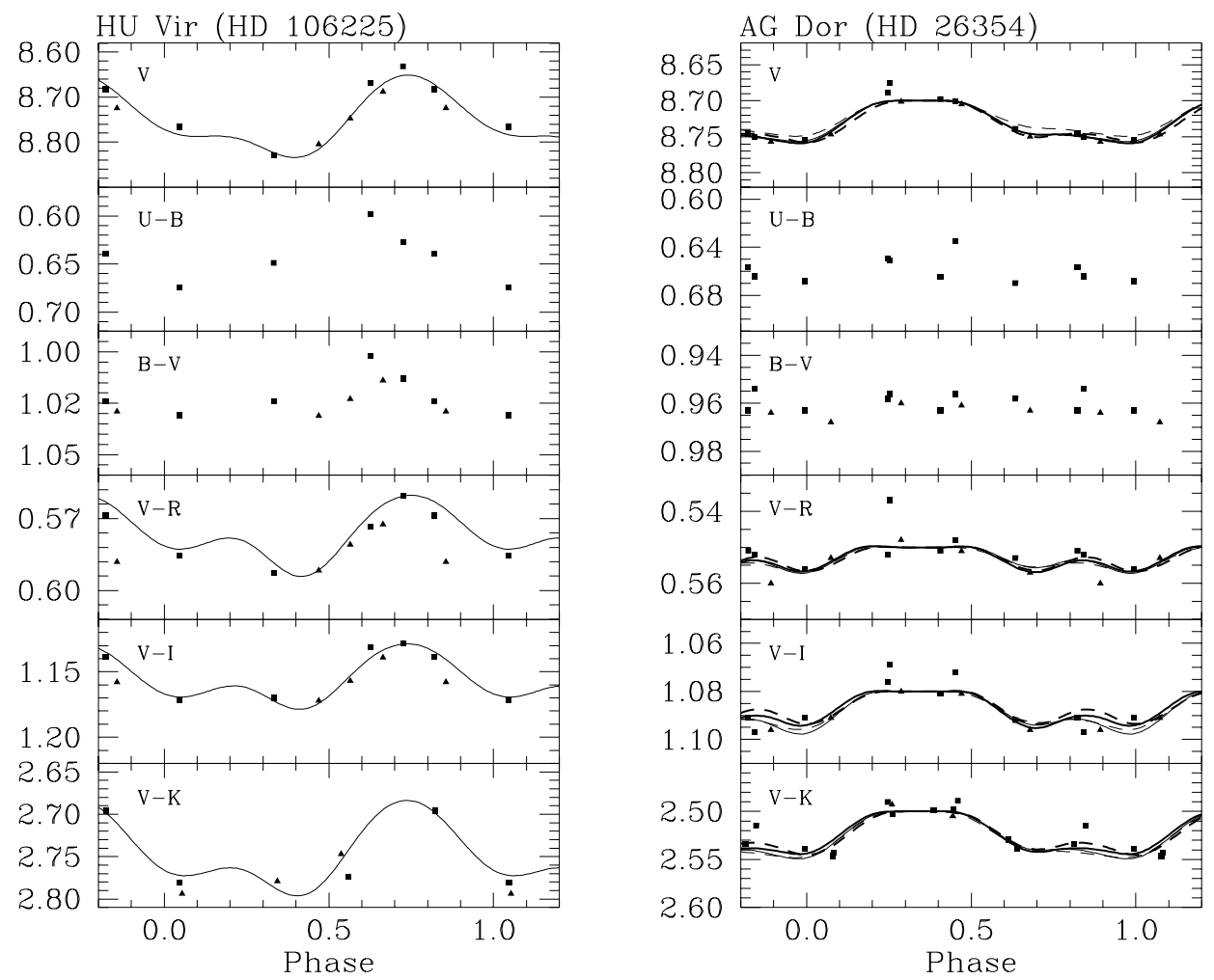

Fig. 5. Light and colour curves for HU Vir and AG Dor. The open squares represent data taken in February 1996, the solid squares data from between December 1996 and beginning of January 1997 and the solid triangles data from the end of January and beginning of February 1997. For AG Dor, the solid lines represent Model 1 (thick) and Model 2 (thin) and the dashed lines Model 3 (thick) and Model 4 (thin). The parameters for each model are given in Tables 4 and 5 for AG Dor and HU Vir respectively.

the errors. Figure 6 shows the distribution of the modulating spots on the surface of HU Vir at four different viewing angles of 0, 90, 180 and 270 degrees. The inclination of the rotation axis of the star is $i=65^{\circ}$, but this is not shown in Fig. 6.

\section{Conclusions}

In this paper, the optical and infrared colour curves of the stars AG Dor and HU Vir have been modelled simultaneously to try to remove the non-uniqueness of the solution of the spot problem, thus obtaining $T_{\text {eff }}$, size (radius) and location (latitude and longitude) of the spots for these two stars. Applying the photometric modelling code SPOTPIC, two modulating spots were found on the photosphere of HU Vir (no attempt at modelling the non-modulating component was made on this star) and other two, together with a non-modulating component which was recreated with a polar spot, on AG Dor in December 96/January 97.

For HU Vir, the light and colour curves were modelled with two spots with temperatures of 4450 and $4050 \mathrm{~K}$. The non-modulating component was not include in the model, because the star was close to its historical maximum brightness. On AG Dor, the spot temperatures were constrained to be between 4300 and $4600 \mathrm{~K}$, for the modulating component, and cooler, viz. $4000 \mathrm{~K}$, for the nonmodulating one. From the photometric data, filling factors

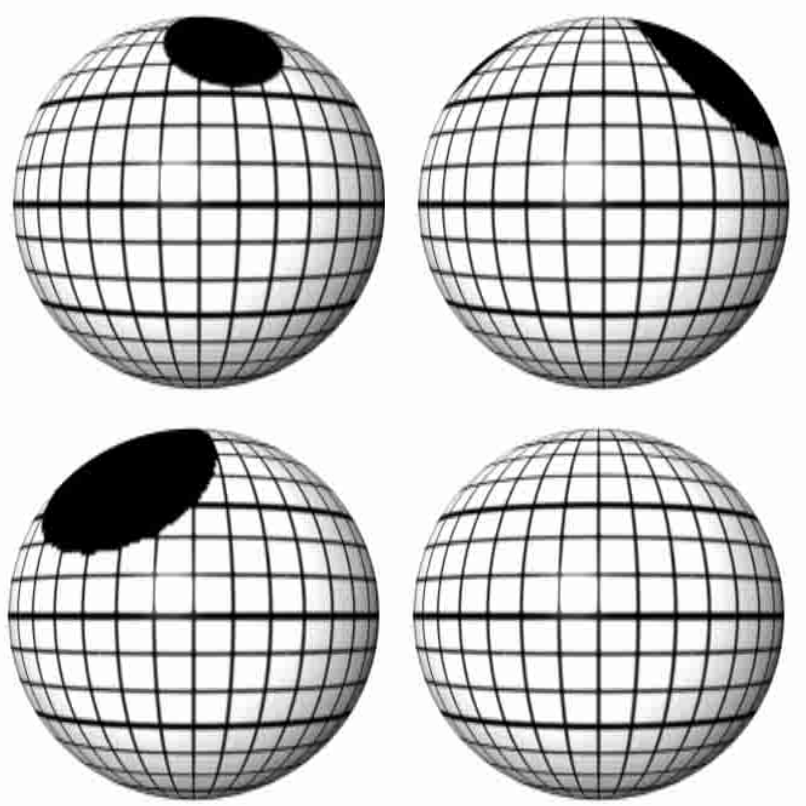

Fig. 6. HU Vir, rotating from right to left, viewed at 0 (upper left), 90 (upper right), 180 (lower left) and 270 (lower right) degrees. Again, only the position on the surface of the star, but not the temperature, of the spot distribution is represented. Grid as in Fig. 1. 
of AG Dor's total coverage of spots were determined to be between 4\% (Model 1) and 7\% (Model 2) for the modulating component, and $26 \%$ for the non-modulating one, which is consistent with the value determined from the spectroscopic data. Therefore, the non-modulating component of spots on AG Dor at this epoch is held to be totally responsible for the presence of the $\lambda 7055 \AA \mathrm{TiO}$ band in the spectra of this $\mathrm{K} 1$ dwarf with a photospheric temperature of $5000 \mathrm{~K}$.

Acknowledgements. PJA acknowledges financial support from Armagh Observatory. MZB was partially supported by the grant 2/1024/21 of Grant Agency for Science.

\section{References}

Amado, P. J. 1997, Ph.D. Thesis, Queen's University, Belfast Amado, P. J., Butler, C. J., \& Byrne, P. B. 1999, MNRAS, 310, 1023

Amado, P. J., Doyle, J. G., \& Byrne, P. B. 2000, MNRAS, 314, 489

Amado, P. J., Zboril, M., Butler, C. J., \& Byrne, P. B. 2001, CoSka, 31, 13

Cutispoto, G. 1996, A\&AS, 119, 281

Cutispoto, G. 1998, A\&AS, 127, 207

Dempsey, R. C., Linsky, J. L., Fleming, T. A., \& Schmitt, J. H. M. M. 1993, ApJS, 86, 599

Drake, S. A., Simon, T., \& Linsky, J. L. 1989, ApJS, 71, 905

Fekel, F. C., Hall, D. S., \& Henry, G. W. 1984, IAU Comm., 27, Inf. Bull. Var. Stars, 2543
Fekel, F. C., Moffet, T. J., \& Henry, G. W. 1986, ApJS, 60, 551

Montes, D., Fernández-Figueroa, M. J., Cornide, M., \& De Castro, E. 1996, A\&A, 312, 221

Neff, J. E., O'Neal, D., \& Saar, S. H. 1992, in Inside the Stars, ed. W. Weiss, ASP Conf. Ser., 40, 193

Neff, J. E., O’Neal, D., \& Saar, S. H. 1995, ApJ, 452, 879

O’Neal, D., Saar, S. H., \& Neff, J. E. 1996, ApJ, 463, 766

Ramsey, L. W. \& Nations, H. L. 1980, ApJ, 239, L121

Schmidt-Kaler, T. 1982, in Landolt-Börnstein, ed. K. Schaifers, \& H. Voigt, vol. 2b (Heidelberg: Springer)

Strassmeier, K. 2001, in Cool Stars, Stellar Systems and the Sun, ed. R. J., García Lopez, R., Rebolo, \& M. R., Zapatero Osorio, Vol. 223, 271

Strassmeier, K. G. 1994, A\&A, 281, 395

Strassmeier, K. G., Bartus, J., Cutispoto, G., \& Rodonò, M. 1997, A\&AS, 125, 11

Strassmeier, K. G., Fekel, F. C., Bopp, B. W., Dempsey, R. C., \& Henry, G. W. 1990, ApJS, 72, 191

Tody, D. 1986, in Instrumentation in Astronomy VI, ed. D. Crawford, vol. 627, Proc. SPIE, 733

Vogt, S. S. 1979, PASP, 91, 616

von der Lühe, O., Solanki, S., \& Reinheimer, T. 1996, in Stellar Surface Structure, International Astronomical Union, ed. K. G. Strassmeier, \& J. L. Linsky (Vienna: Kluwer academic Publishers), Symp. 176, 147

Washüttl, A., \& Strassmeier, K. G. 1995, in Poster Proceedings Stellar Surface Structure, ed. K. Strassmeier, International Astronomical Union (Vienna: Kluwer Academic Publishers), Symp. 176 